\title{
The Risk of Malignancy in Korean Patients with Rheumatoid Arthritis
}

\author{
Hwajeong Lee \\ Division of Rheumatology, Department of Internal Medicine, Arthritis \& Autoimmunity Research Center, Daegu Catholic University \\ School of Medicine, Daegu, Korea.
}

Purpose: To investigate the overall cancer risk and risk for specific cancers in rheumatoid arthritis (RA) patients in Korea by comparing cancer incidence between RA patients and the general population.

Materials and Methods: Individuals diagnosed with RA between 1996 and 2009 who underwent treatment at the Daegu Catholic University Medical Center were retrospectively examined. 1885 patients with RA were included in the analyses. Occurrence of cancer and death during follow up was ascertained by linking medical records to the Korean Central Cancer Registry and national death certificates. For comparing cancer incidence between RA patients and general population, standardized incidence ratios (SIR) were calculated. The 95\% confidence intervals (CIs) of SIRs were calculated using the shortcut method introduced by Vandenbroucke.

Results: The total follow-up time was 10218.9 person-years. During follow up, 100 patients (31 men and 69 women) were diagnosed with cancer. Both men and women had greater risks of having malignancy, although cancer risk was greater in men. Men showed increased risks of lung cancer (SIR=5.46, 95\% CI: 2.60-9.36) and leukemia (SIR=16.7, 95\% CI: 1.58-47.9). Women showed increased risks of thyroid cancer (SIR=1.75, 95\% CI: 1.02-2.68), cervical cancer (SIR=3.65, 95\% CI: 1.65-6.42), non-Hodgkin's lymphoma (SIR=6.47, 95\% CI: 2.04-13.4), and gallbladder cancer (SIR=3.87, 95\% CI: 1.01-8.60). Disease-modifying anti-rheumatic drugs usage and cancer were not related: the relative risks of developing malignancy were not elevated for each medicine.

Conclusion: The overall cancer incidence was increased in Korean men and women with RA. Increased risk of specific malignancy differed according to sex.

Key Words: Rheumatoid arthritis, malignancy, cancer, incidence, standardized incidence ratio

\section{INTRODUCTION}

Rheumatoid arthritis (RA) is an inflammatory disease with a long disease duration. RA patients undergo long-term disease-modifying anti-rheumatic drugs (DMARDs) therapy and may suffer from various comorbidities, such as malignancy,

Received: December 7, 2018 Revised: December 27, 2018

Accepted: December 28, 2018

Corresponding author: Hwajeong Lee, MD, Division of Rheumatology, Department of Internal Medicine, Arthritis and Autoimmunity Research Center, Daegu Catholic University School of Medicine, 33 Duryugongwon-ro 17-gil, Nam-gu, Daegu 42472, Korea.

Tel: 82-53-650-4214, Fax: 82-53-629-8248, E-mail: hlee@cu.ac.kr

-The author has no potential conflicts of interest to disclose.

(C) Copyright: Yonsei University College of Medicine 2019

This is an Open Access article distributed under the terms of the Creative Commons Attribution Non-Commercial License (https://creativecommons.org/licenses/ by-nc/4.0) which permits unrestricted non-commercial use, distribution, and reproduction in any medium, provided the original work is properly cited. cardiovascular disease, and lung disease. Several epidemiologic studies have suggested an association between RA and malignancy. ${ }^{1-4}$ Nevertheless, it remains unclear whether the overall cancer incidence rate is increased in RA patients: several studies have shown an increased overall cancer risk in RA patients, although some studies have reported no increase in overall cancer incidence. ${ }^{5-7}$

Regarding site-specific cancers, several studies have reported that the incidence of lung cancer and lymphoma is greater in RA patients. ${ }^{2,4,8}$ Simon, et al. ${ }^{4}$ performed a meta-analysis of the incidence of malignancy in adult patients with RA and found that RA patients had increased risks of lymphoma and lung cancer and decreased risks of colorectal and breast cancers. There are no consistent findings on the risk of cervical cancer, prostate cancer, and melanoma.

The incidences of specific malignancies are affected by a patient's region, race, age, sex, and times. In men, the five lead- 
ing primary sites of malignancy were the stomach, lung, liver, colon and rectum, and bladder in 1999-2001. ${ }^{9}$ Since then, the number of cases of colon cancer has increased, with colon cancer becoming the third most common cancer in $2015 .{ }^{10} \mathrm{In}$ women, the five leading primary sites of malignancy were the stomach, breast, colon and rectum, uterine cervix, and lung in 1999-2001. ${ }^{9}$ Since then, the numbers of cases of thyroid cancer have increased rapidly, and thyroid cancer became the most common cancer in $2015 .{ }^{10}$ In Korea, studies have been conducted on the incidence of malignancies in RA patients using biologic DMARDs; however, there has been no study on the risk of malignancy in RA patients versus the general population. ${ }^{11,12}$

In this study, we investigated the overall cancer risk, as well as the risks for specific cancer types, in RA patients in Korea by comparing the incidence of cancer among RA patients versus that in the general population.

\section{MATERIALS AND METHODS}

\section{Study population}

RA patients diagnosed with the disease between 1996 and 2009 and who underwent treatment at the Daegu Catholic University Medical Center were selected retrospectively as eligible subjects for the current study. Patients with missing data regarding their personal identification number or the date of RA diagnosis were excluded from the study population. The patients whose malignancies were confirmed before the diagnosis of RA were also excluded from the eligible study population to form a cancer-free cohort. Finally, 1885 patients with RA who were $\geq 19$ years of age at the time of diagnosis of the disease were included in the analyses. The study was approved by the Daegu Catholic University Medical Center Institutional Review Board (IRB No. CR-11-017).

\section{Identification of cancer cases and statistical analysis}

The occurrence of cancer and death during the follow-up period among the study participants was ascertained via the linkage of medical records to the Korean Central Cancer Registry and national death certificates. In Korea, patients diagnosed with cancer using imaging study or tissue biopsy are registered in the Korea Central Cancer Registry. The code C00-97 of the tenth version of the International Classification of Diseases (ICD-10) was used for the identification of cancer cases by site. In order to determine the follow-up period, the starting points were defined as the date of RA diagnosis. The end-points were defined as the earliest date among the following: the date of cancer registration, date of death, and December 31, 2009 (the end of the observation period). For the comparison of cancer incidence in RA patients with that in the general population of Korea, we calculated the standardized incidence ratio (SIR), which is defined as the ratio of the observed number to the expected number of cancer cases. The expected number of cancer cases was estimated using the national cancer incidence rates [age-specific cancer incidence rates for 18 age groups (i.e., 15-19 years, 20-24 years, 25-29 years, and groups of 5 -year categories up to $\geq 85$ years) by sex] for 1999, 2004, and 2009 based on the Korea National Cancer Incidence Database. ${ }^{13}$ The $95 \%$ confidence intervals (CIs) of SIR were calculated using the following shortcut method introduced by Vandenbroucke: ${ }^{14}$

$$
\frac{(\sqrt{\text { Observed number of cancer cases }} \pm 1.96 \times 0.5)^{2}}{\text { Expected number of cancer cases }} .
$$

We conducted separate analyses for men and women as they differed in their cancer risks. Site-specific SIRs were calculated for seven leading cancer sites. The differences in the use of medication between the no-cancer and cancer groups were assessed using Fisher's exact tests or chi-square tests; missing values $(\mathrm{n}=3)$ were excluded from the analysis. Cox proportional hazards models were used to calculate the relative risks (RRs) of cancer according to the use of medication after adjusting for age and/or sex. All statistical analyses were performed by the IBM SPSS Statistics 19.0 (IBM Corp., Armonk, NY, USA) and the Microsoft Office Excel 2007 (Microsoft Corp., Redmond, WA, USA).

\section{RESULTS}

\section{Risk of cancers in patients with RA}

We enrolled 1885 patients with RA who were not previously diagnosed with malignancies. Among the 1885 study participants, men accounted for $15.9 \%$ and women $84.1 \%$. The mean age $( \pm$ SD) of the study participants at the time of diagnosis of RA was $55.8( \pm 11.0)$ and $52.0( \pm 12.8)$ years in men and women, respectively.

The total follow-up time was 10218.9 person-years (1341.8 person-years for men; 8877.1 person-years for women). During the follow-up period from 1996 to 2009, 100 patients were diagnosed with cancer (31 cases for men; 69 cases for women). The seven leading sites of cancer incidence were as follows: for men, lung (10 cases), colon and rectum (5 cases), stomach ( 3 cases), prostate (3 cases), non-Hodgkin's lymphoma (2 cases), bladder ( 2 cases), and leukemia ( 2 cases); for women, thyroid gland (17 cases), stomach (10 cases), breast ( 9 cases), cervix uteri (9 cases), non-Hodgkin's lymphoma (5 cases), gallbladder (4 cases), and colon and rectum (3 cases) (Table 1).

The overall incidence rate of all cancers was 978.6/100000 (2310.3/100000 for men; 777.3/100000 for women). In RA patients, both men and women had greater risks of being diagnosed with malignancy, with the cancer risk greater in men than in women. 
In male RA patients, the SIR of cancer was greatest among patients aged $\geq 65$ years (SIR 2.94, 95\% CI: 1.64-4.62). In female RA patients, the SIR for cancer among patients aged 1534 years was the greatest (SIR 3.88, 95\% CI: 1.01-8.61), while that for patients aged $\geq 65$ years was not significantly different from that of the general population (Table 2).

\section{Specific types of cancers associated with RA}

The risk for specific cancer types in RA patients was evaluated in comparison with the general Korean population. The risk for specific cancer types differed slightly according to sex. Men had greater risks of lung cancer (SIR=5.46, 95\% CI: 2.609.36) and leukemia (SIR=16.7, 95\% CI: 1.58-47.9), while women had increased risks for thyroid cancer ( $\mathrm{SIR}=1.75,95 \% \mathrm{CI}$ : 1.02-2.68), cervical cancer ( $\mathrm{SIR}=3.65,95 \% \mathrm{CI}$ : $1.65-6.42)$, non-
Hodgkin's lymphoma (SIR=6.47, 95\% CI: 2.04-13.4), and gallbladder cancer (SIR=3.87, 95\% CI:1.01-8.60). The SIR was highest for non-Hodgkin's lymphoma (SIR=6.47, 95\% CI: 2.04-13.4) in female RA patients. In both men and women, the risks for the stomach, colon, and rectal cancer were not significantly different than those in the general population (Table 3).

\section{Malignancy and the treatment of RA}

The use of hydroxychloroquine was high at $78.4 \%$ in the no cancer group, with no difference in other DMARDs used between the cancer and no-cancer groups among RA patients (Table 4). No association was noted between DMARDs usage and cancer, and the RR of developing malignancy was not elevated in each medicine (Table 5).

Table 1. Number of Cancer Cases among Rheumatoid Arthritis Patients by Sex during the Study Period

\begin{tabular}{|c|c|c|c|}
\hline \multicolumn{2}{|c|}{ Males ( $n=299$ ) } & \multicolumn{2}{|c|}{ Females ( $n=1586)$} \\
\hline Site of cancer (ICD-10 code) & Cases & Site of cancer (ICD-10 code) & Cases \\
\hline Lung (C33-C34) & 10 & Thyroid gland (C73) & 17 \\
\hline Colon and rectum (C18-C20) & 5 & Stomach (C16) & 10 \\
\hline Stomach (C16) & 3 & Breast (C50) & 9 \\
\hline Prostate (C61) & 3 & Cervix uteri (C53) & 9 \\
\hline Non-Hodgkin's lymphoma (C82-C85, C96) & 2 & Non-Hodgkin's lymphoma (C82-C85, C96) & 5 \\
\hline Bladder (C67) & 2 & Gallbladder (C23-C24) & 4 \\
\hline Leukemia (C91-95) & 2 & Colon and rectum (C18-C20) & 3 \\
\hline Pancreas (C25) & 1 & Liver (C22) & 2 \\
\hline Larynx (C32) & 1 & Ureter (C66) & 2 \\
\hline Kaposi's sarcoma (C46) & 1 & Melanoma of skin (C43) & 2 \\
\hline \multirow[t]{6}{*}{ Ureter (C66) } & 1 & Oral cavity (CO2) & 1 \\
\hline & & Lung (C33-C34) & 1 \\
\hline & & Ovary (C56) & 1 \\
\hline & & Kidney (C64) & 1 \\
\hline & & Bladder (C67) & 1 \\
\hline & & Brain (C71) & 1 \\
\hline All cancer (COO-C97) & 31 & & 69 \\
\hline
\end{tabular}

ICD-10: the tenth version of the International Classification of Diseases.

Table 2. SIR of Malignancy among Rheumatoid Arthritis Patients by Sex and Age Groups

\begin{tabular}{|c|c|c|c|c|c|}
\hline \multirow{2}{*}{ Sex and age group } & \multirow{2}{*}{$\begin{array}{c}\text { No. of patients } \\
(\%)\end{array}$} & \multirow{2}{*}{ Person-years } & \multicolumn{2}{|c|}{ Incidence } & \multirow{2}{*}{$\operatorname{SIR}\left(95 \% \mathrm{Cl}^{*}\right)$} \\
\hline & & & Observed & Expected & \\
\hline Males & $299(15.9)$ & 1341.8 & 31 & 11.6 & $2.68(1.82-3.71)$ \\
\hline \multicolumn{6}{|l|}{ Age at diagnosis (yr) } \\
\hline $15-34$ & $7(2.3)$ & 34.0 & 0 & 0.007 & - \\
\hline $35-64$ & $215(71.9)$ & 1049.1 & 16 & 6.5 & $2.48(1.41-3.84)$ \\
\hline$\geq 65$ & 77 (25.8) & 258.7 & 15 & 5.1 & $2.94(1.64-4.62)$ \\
\hline Females & $1586(84.1)$ & 8877.1 & 69 & 42.0 & 1.64 (1.28-2.05) \\
\hline \multicolumn{6}{|l|}{ Age at diagnosis (yr) } \\
\hline $15-34$ & $162(10.2)$ & 982.9 & 4 & 1.0 & $3.88(1.01-8.61)$ \\
\hline $35-64$ & 1133 (71.4) & 6745.4 & 50 & 31.2 & 1.60 (1.19-2.08) \\
\hline$\geq 65$ & $291(18.4)$ & 1148.8 & 15 & 9.8 & $1.53(0.86-2.41)$ \\
\hline
\end{tabular}

SIR, standardized incidence ratios; $\mathrm{Cl}$, confidence interval.

$*(\sqrt{\text { observed number of cancer cases }} \pm 1.96 \times 0.5)^{2} /$ expected number of cancer cases. ${ }^{14}$ 
Table 3. SIR of Seven Leading Cancer Sites among Rheumatoid Arthritis Patients by Sex

\begin{tabular}{|c|c|c|c|}
\hline \multirow{2}{*}{ Sex and cancer site } & \multicolumn{2}{|c|}{ Incidence } & \multirow{2}{*}{$\operatorname{SIR}\left(95 \% \mathrm{CI}^{*}\right)$} \\
\hline & Observed & Expected & \\
\hline \multicolumn{4}{|l|}{ Males ( $n=299$ ) } \\
\hline Lung (C33-C34) & 10 & 1.8 & $5.46(2.60-9.36)$ \\
\hline Colon and rectum (C18-C20) & 5 & 1.6 & $3.07(0.97-6.35)$ \\
\hline Stomach (C16) & 3 & 2.6 & $1.17(0.22-2.88)$ \\
\hline Prostate (C61) & 3 & 0.7 & $4.53(0.85-11.1)$ \\
\hline Non-Hodgkin's lymphoma (C82-C85, C96) & 2 & 0.2 & $9.36(0.88-26.8)$ \\
\hline Bladder (C67) & 2 & 0.3 & $6.29(0.59-18.0)$ \\
\hline Leukemia (C91-95) & 2 & 0.1 & $16.7(1.58-47.9)$ \\
\hline \multicolumn{4}{|l|}{ Females ( $n=1586)$} \\
\hline Thyroid gland (C73) & 17 & 9.7 & $1.75(1.02-2.68)$ \\
\hline Stomach (C16) & 10 & 4.9 & $2.03(0.97-3.48)$ \\
\hline Breast (C50) & 9 & 6.8 & $1.33(0.60-2.34)$ \\
\hline Cervix uteri (C53) & 9 & 2.5 & $3.65(1.65-6.42)$ \\
\hline Non-Hodgkin's lymphoma (C82-C85, C96) & 5 & 0.8 & $6.47(2.04-13.4)$ \\
\hline Gallbladder etc. (C23-C24) & 4 & 1.0 & $3.87(1.01-8.60)$ \\
\hline Colon and rectum (C18-C20) & 3 & 4.6 & $0.66(0.12-1.61)$ \\
\hline
\end{tabular}

SIR, standardized incidence ratios; $\mathrm{Cl}$, confidence interval.

$*(\sqrt{\text { observed number of cancer cases }} \pm 1.96 \times 0.5)^{2} /$ expected number of cancer cases. ${ }^{14}$

Table 4. Medication Use among Rheumatoid Arthritis Patients by Cancer during the Study Period

\begin{tabular}{lccc}
\hline & $\begin{array}{c}\text { No cancer } \\
\text { (n=1785) } \\
\mathbf{n ( \% )}\end{array}$ & $\begin{array}{c}\text { Cancer } \\
\text { (n=100) } \\
\mathbf{n}(\%)\end{array}$ & p value* $^{*}$ \\
\hline Methotrexate & $1621(90.8)$ & $87(87.0)$ & 0.20 \\
Hydroxychloroquine & $1400(78.4)$ & $68(68.0)$ & 0.01 \\
Sulfasalazine & $1000(56.0)$ & $59(59.0)$ & 0.56 \\
Leflunomide & $268(15.0)$ & $13(13.0)$ & 0.58 \\
Azathioprine & $62(3.5)$ & $3(3.0)$ & 0.80 \\
Tacrolimus & $115(6.4)$ & $3(3.0)$ & 0.17 \\
Cyclosporine & $104(5.8)$ & $8(8.0)$ & 0.37 \\
Bucillamine & $290(16.3)$ & $19(19.0)$ & 0.47 \\
Biologic DMARDs & $134(7.5)$ & $6(6.0)$ & 0.58 \\
NSAIDs & $1751(98.1)$ & $97(97.0)$ & 0.44 \\
Steroids ${ }^{\dagger}$ & $1730(97.1)$ & $96(96.0)$ & 0.54 \\
\hline
\end{tabular}

DMARDs, disease-modifying anti-rheumatic drugs; NSAIDs, nonsteroidal anti-inflammatory drugs.

${ }^{*}$ Calculated by chi-square tests or Fisher's exact tests, ${ }^{\dagger}$ Missing values $(n=3)$ were excluded from the analysis.

\section{DISCUSSION}

Medical records at a single tertiary hospital from 1996 through 2009 were reviewed, and 100 patients were diagnosed with cancer from 1885 RA patients. We compared the incidence of cancer according to sex because cancer incidence in the general population differs according to sex..$^{9,10,13}$ In this study, male and female RA patients had increased risks of malignancy, and notably male patients showed a greater risk (SIR 2.68, 95\% CI: 1.82-3.71) than female patients (SIR 1.64, 95\% CI:
1.28-2.05). Male RA patients aged $\geq 65$ years showed the highest incidence rate of cancer, while the incidence of cancer was highest in female RA patients aged $\leq 34$ years. Chen, et al. ${ }^{3}$ showed a slightly increased overall cancer risk in patients with RA (SIR 1.23, 95\% CI: 1.22-1.23), and the risk was similar between women and men in Taiwanese patients with RA. On the other hand, Parikh-Patel, et al. ${ }^{1}$ reported that the overall cancer risk in men was similar to that observed in the general population. The overall cancer risk in women was $15 \%$ lower than that of the general California population. Although individual studies have not shown consistent results, Simon, et al. ${ }^{4}$ recently performed a meta-analysis and revealed that patients with RA have a $10 \%$ increase in overall malignancy risk compared with the general population.

The increased risk of hematological malignancies in RA patients is well known, and notably several studies have reported an increase in risk of lymphoma in patients with RA. ${ }^{8}$ In our study, seven lymphoid malignancies (2 men and 5 women) were identified during the follow-up period. The SIR of lymphoid malignancies was increased in female RA patients (SIR 6.47, 95\% CI: 2.04-13.4), although the difference was not significant in men (SIR 9.36, 95\% CI: 0.88-26.8). During the follow-up period, two leukemia cases were identified in male RA patients, although no myeloid malignancy was observed in female RA patients. The SIR of leukemia was increased in male RA patients (SIR 16.7, 95\% CI: 1.58-47.9). Similar results have been reported in previous studies. Lin, et al. ${ }^{15}$ reported a significantly higher incidence of both lymphoid and myeloid malignancies in male RA patients compared with that in RAfree patients in Taiwan. In female RA patients, a significantly increased overall incidence risk was noted in lymphoid malig- 
Hwajeong Lee

Table 5. RRs* and 95\% Cls of Cancer among Rheumatoid Arthritis Patients according to Medication Use

\begin{tabular}{lccc}
\hline & Total (n=1885), RR (95\% Cl) & Male (n=299), RR (95\% Cl) & Female (n=1586), RR (95\% Cl) \\
\hline Methotrexate & $0.73(0.41-1.33)$ & $0.98(0.37-2.57)$ & $0.64(0.30-1.34)$ \\
Hydroxychloroquine & $0.81(0.53-1.24)$ & $0.78(0.35-1.73)$ & $0.79(0.48-1.31)$ \\
Sulfasalazine & $1.01(0.68-1.51)$ & $0.98(0.46-2.09)$ & $1.02(0.63-1.64)$ \\
Leflunomide & $0.86(0.48-1.55)$ & $0.71(0.21-2.35)$ & $0.93(0.48-1.83)$ \\
Azathioprine & $0.65(0.21-2.07)$ & $1.51(0.36-6.37)$ & $0.32(0.05-2.32)$ \\
Tacrolimus & $0.57(0.18-1.80)$ & - & $0.82(0.26-2.61)$ \\
Cyclosporine & $1.11(0.54-2.29)$ & $0.50(0.07-3.72)$ & $1.35(0.62-2.97)$ \\
Bucillamine & $0.83(0.50-1.36)$ & $0.67(0.26-1.74)$ & $0.89(0.49-1.60)$ \\
Biologic DMARDs & $0.93(0.40-2.14)$ & - & $1.08(0.47-2.51)$ \\
NSAIDs & $0.48(0.15-1.54)$ & $0.33(0.08-1.39)$ & $0.88(0.12-6.42)$ \\
Steroids & $0.50(0.19-1.37)$ & $0.89(0.12-6.73)$ & $0.48(0.15-1.54)$
\end{tabular}

RR, relative risk; Cl, confidence interval; DMARDs, disease-modifying anti-rheumatic drugs; NSAIDs, Nonsteroidal anti-inflammatory drugs.

${ }^{*}$ Cox proportional hazards models were used to calculate the RRs of cancer according to medication use, after adjusting for age and/or sex, ${ }^{\dagger}$ Missing values $(n=3)$ were excluded from the analysis.

nancies, although no significant increase was noted in myeloid malignancies. The incidence of malignant lymphoma was significantly increased in both male and female RA patients compared to that in the general population in a Japanese study. ${ }^{2}$ Several possible reasons have been attributed to the increased risk of malignant lymphomas in RA patients. For instance, underlying inflammation, the existence of shared genetic risk factors, and an immunosuppressive treatment have been suggested as risk factors for RA-associated lymphomas. Recent studies have indicated an association between disease severity and lymphoma risk in RA. ${ }^{15-17}$ Only a few studies have shown that RA patients have an increased risk of myeloid malignancies. Some cases have reported that myeloid malignancies could be caused by immune-modulating treatments, such as azathioprine or methotrexate, in autoimmune diseases. ${ }^{18,19}$

In our study, lung cancer occurred in 10 of 299 male RA patients and in one out of 1586 female patients. The risk of lung cancer was elevated in male RA patients (SIR 5.46, 95\% CI: 2.60-9.36). The incidence of lung cancer in RA patients is known to be elevated in comparison to that in the general population. Simon, et al. ${ }^{4}$ reported that RA patients have an increased risk of lung cancer compared with the general population. The SIRs for lung cancer ranged from 1.36 to 2.9. A retrospective cohort study conducted in RA patients in California revealed lung cancer risk to be elevated in both male (SIR=1.65, 95\% CI:1.49-1.81) and female (SIR=1.28, 95\% CI: 1.19-1.38) RA patients. ${ }^{1}$ While no data were obtained on smoking in this study, other studies have reported smoking rates among the general population in the United States. In 2011, an estimated $19 \%$ of adults were current smokers, with a smoking prevalence of $21.6 \%$ among men and $16.5 \%$ among women. ${ }^{20}$ In our study, the incidence of lung cancer was significantly higher in male RA patients. This result seems to be related to the high smoking rate among men in Korea. According to the report of the Ministry of Health and Welfare in
Korea, $27.2 \%$ of adults aged $>19$ years were smokers, and the smoking rate of Korean men was $46.9 \%$ in 2009. In the study of the Korean Longitudinal Study on Health and Aging (KLoSHA), the prevalences of ever-smoker and current-smoker women were $8.5 \%$ (95\% CI: 5.8-11.2) and 3.9\% (95\% CI: 2.0 5.7), respectively. ${ }^{21}$ In our study, eight of 11 lung cancer patients were smokers. Of the 11 patients, seven reported emphysema, and four had interstitial lung disease. In a South European population study, the 7 patients who developed lung cancer were men, smokers, and aged $>55$ years with a longstanding disease. The authors proposed that variables of being male, increasing age, previous use of cytotoxic, excluding methotrexate, erythrocyte sedimentation rate, and white blood cell count, were associated with a higher risk of developing cancer. ${ }^{22,23}$

The incidence of thyroid cancer was increased in female RA patients compared to that in the general population. In previous studies, the incidence of thyroid cancer was not significantly different from that in the general population..$^{1-3}$ It is difficult to generalize whether the incidence of thyroid cancer is higher in female RA patients using only the results of this study. In Korea, the incidence of thyroid cancer has rapidly increased in the general population, with increasing numbers of people undergoing thyroid cancer screening by ultrasonography. In 2014, the overdiagnosis of thyroid cancer was an issue. ${ }^{24}$ RA patients frequently visit hospitals, which increases the chances of receiving thyroid cancer screening, which could have served as a bias in the study.

In this study, the risk of cervical cancer was greater in RA patients than in the general population (SIR $=3.65,95 \% \mathrm{CI}$ : 1.65-6.42). Previous studies in Taiwan, California, and Japan did not show an increased incidence of cervical cancer in female RA patients, ${ }^{1-3}$ although another study reported an increased risk for cervical cancer. Kim, et al..$^{25}$ studied the risk of high-grade cervical dysplasia and cervical cancer in women with systemic inflammatory disease, including inflammatory 
bowel disease, psoriasis, RA, and systemic lupus erythematosus. Hazard ratios [HRs (95\% CIs)] for high-grade cervical dysplasia and cervical cancer were 1.49 (95\% CI: 1.11-2.00) in RA patients. Wadstrom, et al. ${ }^{26}$ reported that biologic-naïve women with RA were at greater risk of cervical intraepithelial neoplasia (CIN) grade 1 (HR 1.53, CI: 1.23-1.89) and CIN 2-3 (HR 1.39, CI: 1.16-1.66), but not of invasive cervical cancer (HR 1.09, CI: 0.71-1.65), compared with the general population. RA patients are more likely to have persistent human papillomavirus (HPV) due to poor immune competence, which increases the risk of cervical cancer. Increased cervical cancer incidence may also be associated with systemic inflammation and immunosuppressant or the use of steroid. Although the efficacy of HPV vaccine has not been proven in patients with RA, young women with RA should consider a HPV vaccination, because the HPV vaccination is highly effective in preventing cervical cancer in the general population. ${ }^{25-27}$

This study has both limitations and strengths. Because this study was a retrospective observational study in a single center, only limited information is available and the sample size is small. For example, we could not evaluate the relationship between cancer development and RA disease activity. However, information on the diagnosis and treatment period of RA, cancer diagnosis date, and information on the malignancy site has high accuracy. Because the enrolled patients were diagnosed with RA and underwent DMARDs therapy at our hospital, the date of cancer diagnosis and the cancer site were confirmed on records of the Korean Central Cancer Registry and national death certificates along with the medical chart review. In addition, some detailed information about cancer patients was also available through a chart review, and the presence of smoking history or an underlying lung disease was also confirmed in patients with lung cancer.

Previous studies on the development of cancer in patients with RA have been conducted in several countries. This is the first study to compare the incidence of cancer among patients with RA to those of the general population in Korea. The results of this study are expected to be used as the baseline assessment of malignancy among Korean RA patients.

In conclusion, the overall incidence of cancers in Korean RA patients was increased in both male and female patients. The incidences of lung cancer and leukemia were increased in male RA patients, and the incidences of thyroid cancer, cervical cancer, non-Hodgkin's lymphoma, and gallbladder cancer were increased in female RA patients.

\section{ORCID iD}

Hwajeong Lee https://orcid.org/0000-0002-1592-7314

\section{REFERENCES}

1. Parikh-Patel A, White RH, Allen M, Cress R. Risk of cancer among rheumatoid arthritis patients in California. Cancer Causes Control 2009;20:1001-10.

2. Yamada T, Nakajima A, Inoue E, Tanaka E, Taniguchi A, Momohara S, et al. Incidence of malignancy in Japanese patients with rheumatoid arthritis. Rheumatol Int 2011;31:1487-92.

3. Chen YJ, Chang YT, Wang CB, Wu CY. The risk of cancer in patients with rheumatoid arthritis: a nationwide cohort study in Taiwan. Arthritis Rheum 2011;63:352-8.

4. Simon TA, Thompson A, Gandhi KK, Hochberg MC, Suissa S. Incidence of malignancy in adult patients with rheumatoid arthritis: a meta-analysis. Arthritis Res Ther 2015;17:212.

5. Hashimoto A, Chiba N, Tsuno H, Komiya A, Furukawa H, Matsui $\mathrm{T}$, et al. Incidence of malignancy and the risk of lymphoma in Japanese patients with rheumatoid arthritis compared to the general population. J Rheumatol 2015;42:564-71.

6. Huang WK, Chiou MJ, Kuo CF, Lin YC, Yu KH, See LC. No overall increased risk of cancer in patients with rheumatoid arthritis: a nationwide dynamic cohort study in Taiwan. Rheumatol Int 2014;34:1379-86.

7. Kim YJ, Shim JS, Choi CB, Bae SC. Mortality and incidence of malignancy in Korean patients with rheumatoid arthritis. J Rheumatol 2012;39:226-32.

8. Baecklund E, Askling J, Rosenquist R, Ekbom A, Klareskog L. Rheumatoid arthritis and malignant lymphomas. Curr Opin Rheumatol 2004;16:254-61.

9. Shin HR, Won YJ, Jung KW, Kong HJ, Yim SH, Lee JK, et al. Nationwide cancer incidence in Korea, 1999 2001; first result using the national cancer incidence database. Cancer Res Treat 2005; 37:325-31.

10. Jung KW, Won YJ, Kong HJ, Lee ES. Cancer statistics in Korea: incidence, mortality, survival, and prevalence in 2015. Cancer Res Treat 2018;50:303-16.

11. Cho SK, Lee J, Han M, Bae SC, Sung YK. The risk of malignancy and its incidence in early rheumatoid arthritis patients treated with biologic DMARDs. Arthritis Res Ther 2017;19:277.

12. Jung SM, Kwok SK, Ju JH, Park YB, Park SH. Risk of malignancy in patients with rheumatoid arthritis after anti-tumor necrosis factor therapy: results from Korean National Health Insurance claims data. Korean J Intern Med 2017 Nov 29 [Epub]. https://doi. org/10.3904/kjim.2016.374.

13. Korea Central Cancer Registry, National Cancer Center. Annual report of cancer statistics in Korea in 2014. Ministry of Health and Welfare; 2016.

14. JP V. A shortcut method for calculating the 95 percent confidence interval of the standardized mortality ratio. Am J Epidemiol 1982;115:303-4.

15. Lin YC, Chou HW, Tsai WC, Yen JH, Chang SJ, Lin YC. The agerisk relationship of hematologic malignancies in patients with rheumatoid arthritis: a nationwide retrospective cohort study. Clin Rheumatol 2015;34:1195-202.

16. Smedby KE, Baecklund E, Askling J. Malignant lymphomas in autoimmunity and inflammation: a review of risks, risk factors, and lymphoma characteristics. Cancer Epidemiol Biomarkers Prev 2006;15:2069-77.

17. Baecklund E, Iliadou A, Askling J, Ekbom A, Backlin C, Granath F, et al. Association of chronic inflammation, not its treatment, with increased lymphoma risk in rheumatoid arthritis. Arthritis Rheum 2006;54:692-701.

18. Tokuhira M, Kimura Y, Nemoto T, Sagawa M, Tomikawa T, Sakai $\mathrm{R}$, et al. Therapy-related myeloid neoplasm in methotrexate-associated lymphoproliferative disease in a rheumatoid arthritis patient. J Clin Exp Hematop 2014;54:137-41.

19. Alexson E, Brandt KD. Acute leukemia after azathioprine treat- 
ment of connective tissue disease. Am J Med Sci 1977;273:335-40

20. Centers for Disease Control and Prevention. Current cigarette smoking among adults - United States, 2011. MMWR Morb Mortal Wkly Rep 2012;61:889-94.

21. Kim SK, Park JH, Lee JJ, Lee SB, Kim TH, Han JW, et al. Smoking in elderly Koreans: prevalence and factors associated with smoking cessation. Arch Gerontol Geriatr 2013;56:214-9.

22. Abásolo L, Júdez E, Descalzo MA, González-Alvaro I, Jover JA, Carmona L. Cancer in rheumatoid arthritis: occurrence, mortality, and associated factors in a South European population. Semin Arthritis Rheum 2008;37:388-97.

23. Khurana R, Wolf R, Berney S, Caldito G, Hayat S, Berney SM. Risk of development of lung cancer is increased in patients with rheumatoid arthritis: a large case control study in US veterans. J Rheu- matol 2008;35:1704-8

24. Ahn HS, Kim HJ, Welch HG. Korea's thyroid-cancer "epidemic"-screening and overdiagnosis. N Engl J Med 2014;371:1765-7.

25. Kim SC, Glynn RJ, Giovannucci E, Hernández-Díaz S, Liu J, Feldman S, et al. Risk of high-grade cervical dysplasia and cervical cancer in women with systemic inflammatory diseases: a population-based cohort study. Ann Rheum Dis 2015;74:1360-7.

26. Wadström H, Frisell T, Sparén P, Askling J. Do RA or TNF inhibitors increase the risk of cervical neoplasia or of recurrence of previous neoplasia? A nationwide study from Sweden. Ann Rheum Dis 2016;75:1272-8.

27. Wilton KM, Matteson EL. Malignancy incidence, management, and prevention in patients with rheumatoid arthritis. Rheumatol Ther 2017;4:333-47. 